Voix et Images

voixetimages

\title{
Fernand Ouellette : La lumière sous l'abîme
}

\section{Fernand Ouellette}

Volume 5, numéro 3, printemps 1980

\section{Fernand Ouellette}

URI : https://id.erudit.org/iderudit/200227ar

DOI : https://doi.org/10.7202/200227ar

Aller au sommaire du numéro

\section{Éditeur(s)}

Les Presses de l'Université du Québec

\section{ISSN}

0318-9201 (imprimé)

1705-933X (numérique)

Découvrir la revue

Citer cet article

Ouellette, F. (1980). Fernand Ouellette : La lumière sous l'abîme. Voix et Images, 5(3), 483-495. https://doi.org/10.7202/200227ar d'utilisation que vous pouvez consulter en ligne.

https://apropos.erudit.org/fr/usagers/politique-dutilisation/ 


\section{Fernand Ouellette \\ La lumière sous l'abîme}

Le poème, à travers les sens qu'il met en œuvre, appartient au mouvement perpétuel. Ces tergiversations spatiales, ces fluctuations temporelles, si constantes dans l'œuuve de Ouellette, confèrent à tous les thèmes un dynamisme sans cesse renouvelé. S'aimantant les uns aux autres, ils frôlent sans cesse la contradiction pour bientôt la résoudre en une complémentarité vibrante, toujours à reprendre. L'“à la fois", chez le poète, tend à l'établissement d'un "même", d'une relation nouvelle - mais jamais définitive issue de la fusion des contraires.

Or le poète se situe au commencement avant que le monde connu ne soit. De là son assimilation du monde connu pour le reproposer dans une ouverture, dans l'établissement de nouvelles relations de convenance fondées sur le semblable, mais qui échappent à la logique, à la continuité: relations médiatisées par la métaphore dans le faire d'un objet unique. Et ce nouvel univers n'a d'autre fin que sa propre existence 1 .

Ce passage mouvant de la différence à la simultanéité, de la disjonction à la conjonction, s'effectue ici par une opération unificatrice qui se déroule sur deux plans synchrones. D'une part, il s'agit de concilier la perception et la synthèse du mouvement, sans en immobiliser les diverses directions. D'autre part, on subordonne le « désordre» des dualismes à un ordre ternaire, par la création d'une troisième réalité médiane. La tension entre deux antinomies appelle un effort de l'imaginaire et l'engage à la recherche d'une solution active. Mouvement dialectique du glissement des contraires l'un dans l'autre, de la transmutation des oppositions en une fusion à la fois vibratile et synthétique. Une figure rhétorique, l'oxymore, est particulièrement apte à réaliser cette transformation. Quelques exemples illustrent bien comment, à résorber les dualismes, on aboutit à la logique de la réunion et de l'accord, à l'avènement d'une tierce réalité, tendue et saisissante; du mouvement immobile $^{2}$ à la nuit fulgurante ${ }^{3}$, de la " musique muette 4 » à «l'horizon vertical 5 ", jusqu'à “l'éclair sombre ${ }^{6}$ " et au "midi noir ${ }^{7}$ ", se trame l'expression dense d'un réel renouvelé à partir même des oppositions inhérentes aụ réel. 
Voilà d'ailleurs un des lieux communs les plus féconds de la pensée contemporaine, laquelle n'en finit pas de s'interroger sur la logique qui est à l'œuvre dans les contradictions constitutives de toute représentation du réel. [...] Je me contente de dire que la poésie de Ouellette, parce qu'elle est poésie du c centre", est aussi celle de la tension la plus forte entre les contraires ${ }^{8}$.

La différence, l'écart, le contraste incitent donc l'imaginaire à constituer en poésie une simultanéité, une convenance, une médiation.

La tension engendre le mouvement, la vibration. Ainsi on a maintes fois assisté, au cours de la lecture de «Et nous aimions", aux oscillations suscitées, au niveau thématique tout autant que formel, par le travail conciliateur d'un imaginaire tendu. En effet, qu'il explore les grands thèmes de l'amour et de la mort, du désir et de la chair, du silence et de la parole, de l'abîme et de la profondeur, de la fulgurance et de l'errance, du mal et de la douleur, le poète est emporté par la frénésie et le vertige du mouvement. Omniprésente au monde, dans ce vaste et versatile réseau de signes à déchiffrer, l'écriture se transforme avec lui. Elle passe de la course au ralentissement, de la trépidation au piétinement:

Je piétine parmi les images.

Tant de tableaux tourbillonnent 9 .

La poésie se présente alors comme “[...] une fresque du mouvement, la grande parole du mouvement 10 ". Elle se fait pulsation, secousse, onde, rythme: Elle bouge, oscille, tremble, bondit; elle parcourt l'espace et le temps en des déploiements qui vont du tourbillon à la spirale. "C'est en m'acclimatant à la spirale que je remonte. Alors je peux replonger sans anxiété 11 ». Le verbe s'agite pour éprouver toute agitation, il se métamorphose pour saisir toutes les métamorphoses. Et le poème se donne comme un «mobile» tournoyant dans l'espace - ainsi la parole gravite dans le silence - et comme une oscillation tendue du réel: "la cristallisation impossible d'un mouvement qui ne trouve pas de repos, qui ne se ferme pas, bref qui ne revient pas à l'immobile 12 ". Le poème, “ objet unique", est bien un organisme et une mécanique vivante mise en orbite, en fonction d'un parcours ou d'un cycle toujours recommencé d'existence.

\section{L'unité cherchée}

La similitude et la coïncidence, la ressemblance et le rassemblement se cherchent dans le mouvement, à la fois support et principe d'organisation, force qui préside à l'unité. Ainsi, le poème recrée le monde en un monde unifié; à partir du désordre (celui de la nature, celui des catastrophes contemporaines), il propose un équilibre dynamique. "Si la Parole crée le monde, la parole, et singulièrement celle du poète, la recrée. [...] Là où la Nature se propose aux sens dans un désordre universel, la Poésie reconstitue l'unité. Elle nous restitue le sens profond, universel, enfoui en chacun de nous depuis les origines, ne serait-ce que l'instant d'une fulgurance 13 ». Pour 
parvenir à cette transposition re-créatrice et conciliatrice, le poète doit avoir assimilé en lui le monde et il lui faut retrouver l'harmonie des origines. "L'unité perçue au fond de soi permet de saisir l'unité du Tout 14 ". Le poéme alors est rêvé comme un "avant ", comme une " pré-existence " qui s'ouvrirait soudain, intacte et vive, sur l'être et sur le monde.

L'exploration dans l'ceuvre des grands motifs métaphysiques de l'Un et du Multiple concourt à l'appréhension du Tout, d'une harmonie instable mais saisie soudain, à l'instant de sa réalisation, dans l'utopie ou l'euphorie du poème. Comprenons bien cette impatience et cette fébrilité; non point agitation dispersante mais tension unifiante, lente et énergique à la fois, pulsion d'un centre incandescent. Le poème de Ouellette n'a ni commencement ni fin. Son titre même y est fréquemment inséré et il en constitue un élément interne:

C'est un des traits constants du poème de Ouellette que son absence de chute, corollaire d'une égale absence d'introduction. On est tout de suite plongé dans le vif du sujet et on ne le quitte pas. Du « centre» partout, point de circonférence. Le poème maintient la tension des contraires, ne conclut jamais unilatéralement dans le sens de la joie ou de la détresse 15 .

Toute l'œuvre se montre dans un «à vif » sans issue: à la fois centre et origine, choc et éclat, instant durable ou fragment d'éternité.

\section{L'acte du poème}

Par l'analyse détaillée de “ Et nous aimions ", on a pu déceler de façon pragmatique certains $\mathrm{m}$.écanismes de la poétique de Ouellette et voir comment ils concordaient avec ses conceptions de la praxis de la parole. Ici, le poème retrouve son sens premier de faire. Il provient d'un choix, d'une intervention et d'un acte dérivés du langage et du monde, et ainsi, il agit dans le langage et dans le monde. Mais le drame ou la folie de l'écriture poétique réside en ce que sa lumière même, sa liberté et sa connaissance doivent composer avec le hasard. A l'exemple de la lumière, le poème sait, mais il ne sait pas jusqu'où il sait. “De là une certaine folie, une tension parfois insoutenable. L'écriture ne sait pas qu'elle ne rassemble pas au hasard, mais n'assemble que ce qui la fonde, que ce qui l'approche du dévoilement $16 \%$. Pourtant le poème ne tient pas de la gnose; s'il consent à l'illumination, si parfois, en son cheminement discursif, sa perte même contribue à la découverte, il refuse de s'adonner à la seule divagation ou de s'abandonner à la dérive de l'irrationnel et à ses hasardeuses constellations de sens. Pour reconnaître le pouvoir magique de la parole, le poète sait aussi, avec Baudelaire, qu'«ll y a dans le mot, dans le "verbe" quelque chose de "sacré" qui nous défend d'en faire un jeu de hasard 17 ". Avant de parvenir à la délivrance de l'œuvre, le poète pense, observe, travaille et veille: il œuvre. "C'est pourquoi il faut être en "état de poésie", en état d'agir sur le visible, sens profond, mystère de l'activité poétique, de la parole 
authentique. Cet état d'éveil suppose une conscience totale, la véritable voyance où le regard perce même les arcanes de la Nuit 18". Mise en ordre, acte plénier de conscience, avant la révélation, avant la "transfiguration".

\section{Le nombre d'or et le triangle}

Un autre critère d'organisation du mouvement et de la polyvalence des signes, dans l'œuvre de Ouellette, repose sur la rêverie de thèmes fondamentaux, où l'unité triadique s'investit et se finalise d'elle-même. Nombre d'or, le trois fusionne et unifie; et la poésie résiste aux tensions qu'elle traduit dans la résolution dynamique, par l'imaginaire, d'un mouvement dialectique. Une fois liquidée la lutte contre le dualisme et le manichéisme, la notion d'c axes " ou de "pôles» apparaît chez le poète. «Dès qu'un homme accepte les deux póles de son être, de son essence, il n'y a plus de lutte. Les deux lui sont aussi naturels que le jour et la nuit. C'est l'axe indispensable sans lequel l'homme est un errant 19 ".

De conflictuelle qu'elle était, la poésie devient alors conciliatrice, médiatrice. Parmi ces thèmes essentiels qui effectuent la réconciliation des pôles par l'opération unifiante du ternaire, on retiendra celui du mont en général mais plus spécialement le mont Tabor - et celui du triangle noir de la fermme, a la fois gouffre et sommet, lumière et ténèbre. La valeur synthétique de ces motifs est d'ailleurs confirmée par l'étroite parenté qu'ils entretiennent avec de nombreux aspects élémentaires du symbolisme du triangle 20 .

L'intérêt de ces multiples significations symboliques réside en ce que non seulement elles rejoignent différents éléments de la rêverie matérielle et conceptuelle du poète, mais encore elles épousent le projet fondamental de sa poétique: recherche de l'unité et de l'équilibre par l'exploration systématique des thèmes de la femme, du feu, du soleil et de la lumière, par l'investigation de concepts tels la hauteur, la profondeur et l'abîme, l'espace et le temps, enfin par ce mouvement capital chez lui, à savoir la transition, le passage, la transmutation des réalités antinomiques en une réalité accordée.

\section{Le tabor et ses dérivés imaginaires}

Dans cette perspective, le Tabor sera révalateur d'un ordre fondamental, conciliateur et illuminant. "II n'est pas étonnant que le Tabor que j'ai vu de l'est à l'ouest, de l'orient à l'occident, soit un triangle: symbole de l'expérience spirituelle fondamentale. Le Tabor, comme toute église romane, est dans l'axe est-ouest. Le Tabor demeure le temple fondamental de l'illumination 21 ". Le Tabor soutient le sens fulgurant d'une origine et d'une initiation. Lieu altier d'une certitude lumineuse, sommet où s'abolissent le passé et le futur, il porte la révélation et l'énigme de la connaissance, ces deux caractéristiques attribuées à la lumière et au soleil.

II y a pourtant du Tabor quelque lumière venant d'hier et de demain, 
comme une révelation indéchiffrable

de chêne de grive et de neige 22 .

On notera comment la «révélation indéchiffrable» présente aussi trois éléments - arbre, oiseau, neige - qui appellent la luminosité et le mouvement. En outre, ils accompagnent le parcours de la parole et assistent au jaillissement clair de l'inspiration, cette "transfiguration" de l'être. Plus encore, dans la parfaite consistance de l'oiseau se rencontrent, accomplis et fusionnés, tous les éléments. Tous les mouvements s'épanouissent dans cette synthèse du mouvement qu'est le vol. L'oiseau symbolise alors la spiritualité et l'absolue liberté. Représentation de l'Esprit éclairé et éclaireur, l'oiseau participe d'ailleurs à l'unité de la perfection trinitaire.

A l'exemple de la grotte ${ }^{23}$ ou de la fosse illuminée, la montagne est un abîme d'altitude et de profondeur: hauteur profonde, profondeur haute. Eclairée et éclairante, tout comme l'oiseau, elle stimule l'ascension, et tel l'arbre, déployé aux profondeurs souterraines et dans l'espace aérien, elle harmonise le ciel et la terre. "A la pointe de l'Himalaya le ciel et la terre se synchronisent ${ }^{24}$ ". A son sommet, l'horizontal s'est réalisé en vertical, la matière a atteint la plénitude et l'allègement de l'élévation, bref la pesanteur a pris élan en l'air. Se trouvent alors accordés l'air, la terre et la lumière: un seul (même) lieu et trois substances. "La Montagne" incite encore à l'attente et à la vigilance; par son envergure, elle élève, elle «inspire" le souffle. Sa contemplation allume l'œil et prépare la voix. Vigile du blanc: la pensée (blanche) erre dans l'indicible avant la voyance et la résonnance.

\section{Haut je porte du veilleur le blanchir et cet ceil levé en brûlance contre l'avancée très sonore du souffle ${ }^{25}$.}

De l'inspiration à la révélation s'inscrit une expérience unique de la lumière, de la connaissance et de la poésie (de la connaissance poétique), celle que le poète a si souvent évoquée par le terme de "fulgurance". Alors le Tout devient Un, la disparité se nie dans une similitude ou dans une béatitude qui serait celle du blanc: uniformité, identité, condensation du sens. Transparence du sens fulgurant, éblouissant. A entrer en lumière, l'être devient lumière. Transfiguré, il transfigure. C'est d'ailleurs la signification profonde de la transfiguration du Christ au mont Tabor; à travers l'humanité, la divinité soudain resplendit. Le récit que nous en font les évangélistes recoupe de façon constante des thèmes, parmi les plus obsédants de la poésie de Ouellette:

Son visage resplendit comme le soleil. Matth., XVII, 2. En même temps, ses vêtements devinrent blancs comme la lumière, ou comme la neige. Matth., XVII, 2. Brillants et très blancs comme la neige, tels qu'un foulon sur la terre n'en peut faire d'aussi blancs. Marc, IX, 2. Son vêtement devint blanc fulgurant. Luc, IX, $29^{26 .}$ 


\section{Illumination poétlque at quête mystique}

Ces correspondances entre la transfiguration au mont Tabor - ce triangle illuminant - et la fulgurance reconnue par le poète comme le fondement de sa démarche poétique et exprimée par lui a travers la quasitotalité du répertoire des motifs lumineux (on se rappellera encore l'aube transfigurante) vont permettre de comparer l'expérience poétique à l'illumination qui fonde la quête mystique. «Former, pétrir un poème, c'est aussi former et pétrir son être véritable. Ce n'est qu'un seul acte. Et toute cette recherche n'est pas sans analogie avec la vie mystique elle-même. Du moins cette analogie est d'autant plus sentie qu'on a soi-même des tendances mystiques 27 ". Pourtant la ressemblance même incite à scruter la différence. Si elles partent d'un éblouissement qui conduit au désir d'évélation et de recréation, la vision et la voyance du poète ni ne lui donnent Dieu, ni ne le rendent divin. En même temps que la plénitude et l'intensité créatrice, l'artiste éprouve toujours la "privation" et celle-ci se traduira dans son œuvre par cette brèche ou cette faille qui la rappelleront sans cesse comme cuvre humaine. Dans cette perspective, Fernand Ouellette rejoint de très près la pensée d'Anne Hebert.

Le poème s'accomplit à ce point d'extrême tension de tout l'être créateur, habitant soudain la plénitude de l'instant, dans la joie d'être et de faire [...] Mais toute cuvre, si grande soit-elle, ne garde-t-elle pas en son cœur, un manque secret, une poignante imperfection qui est le signe même de la condition humaine dont l'art demeure une des plus hautes manifestations? [...] L'artiste n'est pas le rival de Dieu. II ne tente pas de refaire la création. II demeure attentif à l'appel du don en lui. Et toute sa vie n'est qu'une longue amoureuse attention à la grâce. II lutte avec l'ange dans la nuit. II sait le prix du jour et de la lumière. II apprend, à l'exemple de René Char, que "La lucidité est la brûlure la plus rapprochée du soleil „28.

En outre, l'acte du poète est tributaire d'un matériau brut et lourd: l'opacité de toute parole, le tain des mots ${ }^{29}$. Dans son épaisseur, le langage se heurte d'abord au poids du monde et ensuite à l'Infini, à l'Absolu qu'il voudrait signifier. Mais le "veilleur» du verbe ne peut pas ne pas dire comme il ne peut pas non plus dire l'indicible. C'est dans cette perspective que Henri Brémond - dont Ouellette a pu s'inspirer dans ses réflexions sur les analogies entre l'expérience poétique et l'expérience mystique - distingue le poète et le mystique:

D'un côté comme de l'autre, il y a une certaine saisie, une possession du réel; sans cela, ni poésie, ni mystique; mais chez le poète, la saisie est plus superficielle que chez le mystique; moins solide, moins unifiante [...] Le poète, en tant que poète, ne peut pas ne pas parler. Là est sa gloire et tout ensemble son irrémédiable faiblesse ${ }^{30}$.

Ainsi, la contemplation du poète - même si elle ne tient pas de la " dissolution lumineuse" ni de l'évasion - est sollicitée par l'action nécessaire sur le langage, par le travail, par la tension du désir. La relation 
qu'il projette d'établir appelle un objet, un monde palpable, un être concret, et alors la poésie se sait du domaine de l'imparfait; elle porte la limite de l'homme et elle atteste son impuissance et son mystère. C'est pourquoi

[...] il ne faut jamais confondre les sphères de la vie mystique et de l'expérience poétique. Je marche peut-être vers un indicible qui est Dieu, mais dans mon expérience actuelle, Dieu, bien que toujours présent, ne me semble pas le terme de mon mouvement intérieur, le pôle de la nouvelle relation que je tente d'établir. En cela je m'éloigne radicalement de l'expérience mystique. [...] je n'appelle pas Infini, Absolu, ce qui a la pesanteur de l'image l'impuissance du mot, la profondeur de la souffrance, l'immensité du soleil. Et je ne me crois pas Dieu, parce que je suis tout simplement en mouvement, dans une direction où je suis entraîné à mon insu par tout le dynamisme et l'énergie d'une unicité qui, à mes yeux, demeure un mystère, une zone inaccessible, un abîme fulgurant ${ }^{31}$.

Dès lors, la poésie s'inscrit dans l'ordre du désir, mais il s'agit d'un ordre impulsif, mouvant, explosif. Le logos entend participer à l'assouvissement de l'éros et le désir même du poème dérive de la déchirure qu'est le désir. Une pulsion perpétuelle se heurte à l'inassouvi, à l'inachevé, au repos inaccessible. Le poème, lieu organique et ouvert de la débâcle, du fracas, de la brûlure, riposte à l'indicible, à l'Infini. Objet profane, geste érotique, il ressort et résulte de la conscience du sacré, du désir de l'Absolu.

Accéder à la poésie, c'est d'une certaine manière atteindre à une région profane de la vie mystique. En ce sens seulement, poésie et mystique sont indissociables. Tout cela se manifeste comme une débâcle: le brûlant désir de l'infini. Tout cela déchire. Le désir freudien est-il autre chose? L'on qualifiera comme on voudra ce désir, il demeurera la source d'une recherche de l'infini. Or la poésie est éminemment une expression de ce désir, un mouvement de l'être vers l'assouvissement, une tension qui explose pour finalement se heurter au mur. Si l'on veut considérer la puissance érotique comme telle, elle est bien cette force luttant contre la mort, dont parlent les freudiens. Et la poésie est la fille de cette puissance érotique ${ }^{32}$.

\section{"Le mont noir"}

Alors que la montagne apparaissait comme le lieu sacré de l'illumination et de la révélation prophétiques, le triangle ou le "mont noir 33 " de la femme opère la synthèse entre le profane et le sacré : prophétie et poésie. L'extase de l'adoration et de la profanation conjuguées, - de l'adoration profanatrice réconcilie le mystique et l'érotique. On sait d'ailleurs, avec Georges Bataille, que "l'effusion mystique est comparable aux mouvements de la volupté physique ${ }^{34}$. Centre, passage, antre, abime profond, profondeur surgie, le sexe-triangle conduit d'un seul mouvement "dans le sombre" et "en lumière». II conjugue l'anéantissement et l'appropriation, la perte et la rencontre, la destruction et la création. Destruction créatrice. Là, l'être s'égare et se trouve; il accomplit simultanément le sacrifice et l'offrande, le 
prodige et le supplice. II connaît en même temps le délire et le délice. Possession. Dé-possession. La brisure du désir se mue alors en une plénitude éclatée qui rappellerait à la fois l'éclatement de la naissance et le choc de la mort. L'instant se condense et explose. " $A$ la base de l'érotisme, nous avons l'expérience d'un éclatement, d'une violence au moment de l'explosion ${ }^{35}$ ». Séisme conciliateur, unifiant. Que cette violence trouve son plein épanouissement extatique sur la montagne ou sa réalisation physique au ventre de la femme permet d'apparenter les deux lieux comme centres de connaissance où l'être, un moment, se dissipe et se recueille dans l'éclair de la fulgurance. Ainsi, la forme triangulaire ${ }^{36}$ conduit à une saisie momentanée de la divinité:

Quel venin te servais-je!

dans ce triangle qui fut plus qu'humain ${ }^{37}$.

Lors de la transfiguration du Christ au mont Tabor, le Dieu fait homme ne se départit pas de son humanité, mais il laisse un instant transparaître sa nature divine. De façon analogue dans l'expérience érotique, l'être éprouve ses limites humaines, mais aussi il appréhende et frôle le sacré, cette prescience fascinée d'un au-delà de l'homme, d'un Absolu:

Très sacré fascine le regard ton pubis comme de grandes orgues silencieuses, une forêt montant vers la montagne lisse ${ }^{38}$.

\section{Corps. Écrit}

Par l'exaltation voluptueuse des sens, la femme séductrice et inspiratrice conduit le poète à la découverte et à l'expression des formes et des sens (significations). L'illumination et l'érotisme transfigurent. Traversée du sombre. Passage du blanc. Ainsi, l'écriture, sur la page, fulgure.

\section{Sans éclat ni rupture} tout le blanc passe

par le sombre des membres ${ }^{39}$.

La tension de l'éros et le conflit qu'elle exprime avec thanatos conduisent au jaillissement du poème, à l'avènement nécessaire du logos. Chez Ouellette, la conscience de l'écriture provient de la conscience même de l'opacité et de l'impatience du corps désirant. Tout le corps écrit et s'écrit. D'ailleurs chaque partie du corps représente la totalité du corps autonome qu'elle traduit. Ainsi chaque poème se donne comme un membre complet (un monde, un microcosme) en lui-même, mais en constante relation avec le corps de l'œuvre (macrocosme); la partie est un tout qui correspond avec le Tout. L'œuvre, corps organisé, propose une forme dynamique, issue d'autres formes mouvantes dont elle s'inspire et dont elle est la manifestation. Ainsi, elle transforme et elle informe:

Je pars donc d'une expérience vécue dans le temps, et, par le poème, je me mets en marche vers l'« innommable "; j'informe un langage, le seul 
qui convienne à la métamorphose qui s'opère au delà ou en deçà de ma conscience. Peu à peu, une forme se dégage dans la mesure où une métamorphose véritable s'accomplit. Et cette forme naturellement indissociable de la métamorphose, donc aussi singulière et unique que celle-ci 40 .

C'est bien ce travail de la métamorphose, de la marche et du désir, au mouvement duquel on aura voulu participer dans cette étude; une mise en œuvre (en forme) qui soit «parcours", trajet, circuit mobile ou errant. Un projet, lui-même mis en marche par cette "imagination du désir " qui fonde la lecture-écriture:

A certaine forme d'être, d'acte et d'objet répond l'épiphanie d'une autre forme. L'essai ébranle le fondement de la mémoire en ce qu'il soumet un projet de totalité axé sur le ou les détails d'un tout. Aussi m'apparaîtil comme l'une des formes privilégiées du désir, de l'aspiration, de l'c inespéré". Il est mu davantage par "l'imagination du désir", par le possible, que par la volonté d'élaborer une synthèse, par le saisissement de ce qui est ${ }^{41}$.

Cette correspondance ou cette coïncidence du fragment entier et ouvert avec l'ensemble de l'œuvre, lui-même total mais toujours expansif, notre lecture projette de la démontrer; d'abord par une adhérence « tactile » et relationnelle au poème et à l'œuvre, voire par une tentative de simulation quasi physique du texte ${ }^{42}$, enfin par l'abondante reproduction de citations textuelles concertantes. De cette manière, si le discours critique demeure toujours second par rapport à l'œuvre qui l'inspire - «[...] de la littérature sur de la littérature, de la littérature au second degré $» 43 \ldots$, il aspire au dévoilement d'un " supplément de sens " ou d'un surplus ou d'un " inattendu ", sans passer par l'agression ou par le «forcement" des sens. La poésie de Ouellette signifie et en même temps parle de la parole. En outre, le poète a longuement mûri, dans ses propres essais, le sens global et les sens nombreux, variables, progressifs de son expérience poétique. Ainsi, à la fois lecteur et écrivant, il s'est situé à ce second degré de la conscience où l'implicite s'éclaire de l'explicite. "Toute lecture poétique ne produit pas nécessairement une isotopie se rattachant à la catégorie du Logos, encore que le poème, objet de langage, comporte toujours cette référence implicite 44 ". Chez Ouellette, le langage fait signe et sens, et il se signifie à lui-même comme forme, matériau et objet. Comme corps signifiant. Dès lors, le discours critique devient ce médiateur (méditatif) second entre le texte et sa propre réflexion. Il propose des significations possibles, peut-être réverbérantes, en engageant un dialogue avec le latent, le sens virtuel de l'œuvre.

[La puissance de l'essayiste] lui vient de sa concentration, de sa méditation, de l'efficacité même de sa parole. Son action n'est pas sensiblement différente de celle du poète. Du poème à l'essai s'opère en quelque sorte un renversement de milieu verbal : glissement d'un pôle à l'autre et recherche, entre le concept et le "son-sens", d'un équilibre fragile. L'essai comme le poème sont des "faires" et par conséquent des « actes $» 45$. 
Acte de présence solidaire à l'œuvre, acte de conscience et d'accompagnement, voilà donc le projet idéal de cette lecture. Qu'on y retrouve çà et là de l'errance, du jeu et du je, du hasard et de la divagation, pourrait répondre à l'attente et au vœu même du poète: «Mon essayiste qui se plaît aux saillies, se soumet à la "combustion verbale», accepte fondamentalement «l'errance»; c'est un être qui, comme le souhaitait Chestov, va «au hasard, les yeux fermés"; c'est un être de la divagation, de l'espace ludique $46 \%$.

La lecture poétique engage le sujet dans un itinéraire sans retour; du fragment à la constellation, il éprouve sans cesse le vertige des sens, des formes et des mouvements. L'œuvre bouge sous ses pas et ainsi elle échappe constamment à l'emprise du désir et de la mémoire. Si le lecteur craint l'anamorphose, la forme qu'il propose doit accueillir les métamorphoses mémes du texte dont il s'informe: miroir mobile qui ne peut saisir que le reflet en mouvement et qui traduit l'impuissance ou les limites de tout regard pris à son propre feu. Lire: ligne du risque, point de vue qui ne s'arrête jamais. Le poème, à travers les sens qu'il inscrit, transgresse le silence et le blanc. La lecture du poème ne peut que tendre à la fragile saisie des lueurs et des rumeurs qui persistent quand se sont tus "la parole inouïe 47 ", le silence sonore ou la musique silencieuse.

1. Fernand Ouellette, "La poésie en son lieu " Liberté, Vol. XVIII, no 6, novembredécembre 1975, p. 39. L'cuvre en prose de Fernand Ouellette comprend les ouvrages suivants: Edgard Varèse, Montréal et Paris, $\mathrm{HMH}$ et Seghers, 1968, 285 p. (EV). Les Actes retrouvés, Montréal, HMH, "Constantes", 1970, 226 p. Depuis Novalis, errance et gloses, Montréal, HMH, «Reconnaissances", 1973, 151 p. (DN). Journal dénoué, Montréal, Les Presses de l'Université de Montréal, "Prix de la revue Etudes françaises", 1974, 246 p. (JD). Tu regardais intensément Geneviève, Montréal, Quinze, “Prose entière", 1978, 184 p. (TRIG). L'œuvre poétique est composée de Poésie, Montréal, l'Hexagone, 1972, 283 p. A moins d'indications contraires, nous utiliserons toujours cette édition. Cette rétrospective regroupe les recueils et les textes suivants: Ces anges de sang (AS), Séquences de l'aile (SA), Radiographies (R), Le Soleil sous la mort (SM), Dans le sombre (DS), Evénements (E), Le Périple (P), La Terre d'oú... (TD), Le Poème et lo poétique (PP). Ici, ailleurs, la lumière, Montréal, L'Hexagone, 1977, 93 p. (IAL) fut publié après la rétrospective. Entre parenthèses, nous indiquons les abréviations utilisées. Au moment où nous terminions cette étude paraissait un autre recueil, $A$ découvert, Québec, les Éditions Parallèles, 1979, 40 p. et un essai intitulé Écrire en notre temps, Montréal, HMH, «Constantes», 1979, 158 p. II n'est pas tenu compte de ces ouvrages dans notre essai.

2. "L'immortalité", DS, p. 208.

3. "L'enfance", ibid., p. 184.

4. "Radiographies du jour I", R, p. 63. “ll y a une sorte de musique du regard où les timbres des poètes se répondent. Sans doute faut-il entendre la Grande Musique muette dont parle Lao-Tzeu ". (DN, p. 23).

5. "Jouve", IAL, p. 24. 
6. "L'oiseau noir", ibid., p. 77.

7. "Croule la cime du rêve", AS, p. 14.

8. André Brochu, “Fernand Ouellette, Ici, ailleurs, la lumière», Livres et auteurs québécois 1977, Presses de I'Université Laval, 1978, p. 130. “"L'idée" que la poésie a pour mission de rassembler les contraires, de réaliser dans le langage la "coincidentia oppositorum" de permettre ainsi à l'homme de dépasser ses limites, ou tout au moins de lui donner l'illusion d'un tel dépassement", cette idée n'est assurément pas neuve, puisque M.-J. Lefèbvre [...] la présente comme une opinion répandue, apparentée à la thèse idéaliste de l'art comme pouvoir de révélation de la vérité". (Groupe $\mathrm{M}$, Rhétorique de la poésie, Bruxelles, Éditions Complexe, 1977, p. 82).

9. "L'oppression", IAL, p. 54.

10. EV, p. 131.

11. JD, p. 103.

12. PP, p. 263.

13. DN, p. 143.

14. Loc. cit.

15. André Brochu, op. cit., p. 128.

16. Fernand Ouellette, "Lettre à André Belleau sur la poésie et l'errance", Liberté, Vol. XIV, no 1-2, janvier-avril 1972, p. 7.

17. DN, p. 47.

18. Ibid., p. 114.

19. AR, p. 15.

20. "Le symbolisme du triangle recouvre celui du nombre trois. [...] Le triangle équilatéral symbolise la divinité, l'harmonie, la proportion. Toute génération se faisant par division, l'homme correspond à un triangle équilatéral coupé en deux, c'est-à-dire à un triangle rectangle. Celui-ci, selon l'opinion de Platon dans le Timée, est aussi représentatif de la terre. Cette transformation du triangle équilatéral en triangle rectangle se traduit par une perte d'équilibre. [...] Le triangle est le glyphe du rayon solaire, chez les anciens Mayas, analogue au "petit clou» qui ferme le germe du maïs naissant, lorsqu'il crève la surface du sol. [...] Rattaché au soleil et au maiss, le triangle est doublement symbole de fécondité. [...] Le triangle, la pointe en haut, symbolise le feu et le sexe masculin; la pointe en bas, il symbolise l'eau et le sexe féminin. [...] Outre son importance bien connue dans le Pytagorisme, le triangle est alchimiquement le symbole du feu ; c'est aussi celui du coeur. II faut toujours envisager à ce propos les rapports entre le triangle droit et le triangle inversé, le second étant le reflet du premier: il s'agit des symboles respectifs de la Nature divine du Christ et de sa nature humaine; ce sont encore ceux de la "montagne" et de la "caverne" [...] Le triangle maçonnique signifiait à sa base la Durée et, sur les côtés qui se rejoignent au sommet, Ténébres et Lumière; ce qui composerait le ternaire cosmique. Quant au delta lumineux de la tradition, [...] un tel triangle correspondrait au nombre d'or".

(Dictionnaire des symboles, sous la direction de Jean Chevalier avec la collaboration de Alain Gheerbrant, Paris, Editions Jupiter et Robert Laffont, 1969, p. 768-769).

21. DN, p. 111.

22. «La solitude», DS, p. 214.

23. "Mais la prospection se continue dans une troisième grotte, le nombre "trois" pouvant symboliser ici le lieu de l'opération de l'unité." (DN, p. 70).

24. AR, p. 93

"Le Pélerin gravissait donc la montagne, lieu symbolique de l'expérience religieuse, point de contact entre le ciel et la terre; comme il était, au début du récit, descendu dans la grotte de sa naissance". (DN, p. 86).

25. TD, p. 258.

26. Dictionnaire de la Bible, publié par $F$. Vigouroux avec le concours d'un grand nombre de collaborateurs, Paris, Letouzey et Ané Editeurs, 1912, 5 tomes, p. 2302. 
27. AR, p. 14.

«De plus, il faut bien ajouter qu'il y a une "ressemblance" entre la quête mystique et la recherche poétique. L'une et l'autre proviennent d'une contemplation, d'une illumination, d'un don". (DN, p. 130).

28. Anne Hébert, "Poésie solitude rompue" dans Mystère de la parole, Poèmes, Paris, Seuil, 1960, p. 89.

29. "Les mots souvent se mesurent contre la noirceur du tain".

("Les mots», TD, p. 243)

30. Henri Brémond, Prière et Poésie, Paris, Bernard Grasset, 1926, p. 210. Dans cet ouvrage l'auteur entend démontrer des " analogies de forme et des communautés de mécanisme" ( $p .123$ ) entre l'expérience poétique et celle des contemplatifs; il vise à les éclairer l'une par l'autre. Sur un ton où la polémique se double de l'habileté à manier le paradoxe, il explique les ressemblances et les différences entre les deux quêtes.

"D'où vient cette différence nécessaire? Encore un coup du caractère spécial qui définit l'activité poétique, le poète ayant pour unique objet, non pas, comme le mystique, de s'approprier le don divin, mais de trouver les formules incantatoires par où le courant poétique puisse passer jusqu'à l'Anima du lecteur. Cette union amoureuse qui suit toute expérience mystique normale, l'expérience poétique ne la permet pas. Le poète, en tant que poète, ne s'unit au réel que pour s'en désunir aussitôt. Ce n'est pas là, je vous prie, un paradoxe: ou plutôt, c'en est un, mais pas de mon cru; c'est le paradoxe du poète. Un mystique manqué est un mais pas de mon cru; c'est le paradoxe du poète. Un mystique manquè, disions-nous». (Ibid., p. 213). Du même auteur, lire aussi La Poésie pure, Paris, Bernard Grasset, 1937, 321 p.

31. AR, p. 34.

Dans le même sens, Brémond différencie le réel apprénendé par le mystique - la contemplation de Dieu - de celui auquel aspire le poète: la saisie unifiante et la ré-appropriation du monde et, indirectement, la relation à Dieu.

"Au delà des idées, des images, des sentiments de la sensation - mais, bien entendu, par l'intermédiaire de toutes les activités de surface - la connaissance poétique atteint des réalités. Non pas directement à la réalité souveraine, Dieu lui-même - cela c'est le privilège exclusif de la connaissance mystique - mais à tout le réel créé et sous le réel créé, indirectement à Dieu lui-même..." (Prière et Poésie, p. 148).

32. AR, p. 37.

"Avec le P. de Grandmaison, nous disions au commencement que l'activité poétique est une ébauche naturelle et profane de l'activité mystique". (Henri Brémond, Prière et Poésie, p. 208).

33. "Jouve", IAL, p. 24.

34. Georges Bataille, L'Érotisme, Paris, Union générale d'éditions, "10/18", 1972, p. 246.

35. Georges Bataille, op. cit., p. 102.

36. Le triangle est d'ailleurs la forme que Roland Giguère utilise comme représentation symbolique de l'unité sur la couverture de Poésie de Fernand Ouellette.

37. «Indifférence", DS, p. 173.

38. "Extase», DS, p. 189.

Ct. aussi: "Je te reconnais bien comme la vierge,

ma tourterelle, mon oraison tendre et triste solitaire sur la montagne».

39. “Etincelle», SM, p. 126.

(“Ma fiancée", Ibid., p. 187).

40. Af, p. 31.

41. Fernand Ouellette, "Divagations sur "l'essai" », Etudes littéraires, Vol. 5, no 1, avril 1972, p. 9. 
42. "L'essayiste est un communiant de ce qui pourrait l'annihiler. II veut "penser de tout son corps". » (/bid., p. 11).

43. Jean-Pierre Richard, "Quelques aspects nouveaux de la critique littéraire en France", Le Français dans le monde, no 15, mars 1963, p. 2.

45. Fernand Ouellette, "Divagations sur "l'essai" ", p. 11.

46. Fernand Ouellette, "Divagations sur "l'essai" ", p. 12.

47. Victor Crastre, Poésie et Mystique, Neuchâtel, Éditions de la Baconnière, "Langages", 1966, p. 129. 